Proceedings

\title{
Investigation of Antiproliferative, Apoptotic and Antioxidant Effects of Oleuropein and Vitamin D on Breast Cancer Cell Lines (MCF-7) ${ }^{\dagger}$
}

\author{
Murat Arı ${ }^{1, *}$, Aslihan Karul ${ }^{1}$ and Serhan Sakarya ${ }^{2}$ \\ 1 Department of Biochemistry, Faculty of Medicine, Aydın Adnan Menderes University, Aydın 09000, \\ Turkey; karulab@yahoo.com \\ 2 Department of Infectious Diseases and Clinical Microbiology, Faculty of Medicine, Aydın Adnan \\ Menderes University, Aydın 09000, Turkey; serhansakarya@yahoo.com \\ * Correspondence: muratari60@gmail.com \\ + Presented at the 2nd International Cell Death Research Congress, Izmir, Turkey, 1-4 November 2018. \\ Published: 6 December 2018
}

\begin{abstract}
Breast cancer is the most common female death in women. Different studies are being done for treatment. The aim of this study was to investigate the antiproliferative, apoptotic and antioxidant effects of oleuropein and vitamin D both individually and in combination. Apoptosis is a programmed cell death pattern with genetic regulation that requires energy, allowing cells to be safely removed from the environment after performing their specified biological task. The olive tree has important biological properties and is rich in phenolic substances. These phenolic substances are mainly oleuropein. Oleuropein has many pharmacological effects such as antioxidant, antimicrobial, antiviral, anticancer, antiinflammatory, antiageing. Vitamin D is a hormone the kidneys produce that controls blood calcium concentration and impacts the immune system.
\end{abstract}

Keywords: oleuropein; vitamin D; MCF-7; cytotoxicity; apoptosis; antioxidant

\section{Introduction}

Breast cancer is the most common female and the second most common cause of cancer death in women. In order to find a solution to such a serious health problem, different studies are being done for treatment and the effects of different substances on these diseases are being intensively investigated [1]. The aim of this study was to investigate the antiproliferative, apoptotic and antioxidant effects of oleuropein and vitamin D both individually and in combination. The olive tree has important biological properties and is rich in phenolic substances. These phenolic components are mainly oleuropein. Oleuropein has many pharmacological effects such as antioxidant, antimicrobial, antiviral, anticancer, anti-inflammatory, anti-ageing [2]. Vitamin D is a hormone the kidneys produce that controls blood calcium concentration and impacts the immune system [3].

\section{Materials and Methods}

MCF-7 was maintained in DMEM (DMEM-HA-CAPRICORN) 10\% fetal bovine serum (FBS), 1\% antibiotic (penicillin-streptomycin, ciprofloxacin. Different concentrations of oleuropein and vitamin D were applied on the MCF-7 cell lines to determine the IC50 values using the WST-1 (water-soluble tetrazolium test) colorimetric assay at 24, 48 and $72 \mathrm{~h}$. Cells were treated with 12.5, 25, 50, 100, 200, 400, 600, 800, 1000, 1200, $1600 \mu \mathrm{M}$ concentration of oleuropein for 24 h. 9.4, 18.75, 37.5, 75, 150, 300, 400, 600, 800, 1000, $1200 \mu \mathrm{M}$ concentration of oleuropein for 48 h. 6.25, 12.5, 25, 50,100, 200, 300, 400, $500,600,800 \mu \mathrm{M}$ concentration of oleuropein for $72 \mathrm{~h}$. Cells were treated with 1.95, 3.906, 7.8125, 
$15.62,31.25,62.5,125,250,1000 \mathrm{nM}$ concentration of vitamin D for $24 \mathrm{~h} .0 .975,1.95,3.906,7.8125$, $15.62,31.25,62.5,125,250,500,1000 \mathrm{nM}$ concentration of vitamin D for 48 and $72 \mathrm{~h}$. No significant results at these doses for vitamin $\mathrm{D}$. Therefore, cells were treated $0.25,0.5,1,2,3,4,6,8,12 \mu \mathrm{M}$ concentration of vitamin D for 24, 48 and $72 \mathrm{~h}$. After finding IC50 values of oleuropein and vitamin $\mathrm{D}$ at $48 \mathrm{~h}$, annexin $\mathrm{V}$ and caspase $3 / 7$ kits were used to determine apoptotic effects foroleuropein, vitamin D and oleuropein-vitamin D combination. After, cell lysate were obtained. Firstly, protein level of the cell lysate was determined. Subsequently, TOS (total oxidant status), TAS (total antioxidant status), OSI (oxidative stress index, SOD (superoxide dismutase), GPx (glutathione peroxidase), MDA (malondialdehyde) and NO (nitric oxide) values were determined.

\section{Results}

IC50 dose of oleuropein was calculated $256.1 \mu \mathrm{M}$ at $24 \mathrm{~h}, 247.5 \mu \mathrm{M}$ at $48 \mathrm{~h}, 222.5 \mu \mathrm{M}$ at $72 \mathrm{~h}$. IC50 dose of vitamin D was calculated $2.053 \mu \mathrm{M}$ at $48 \mathrm{~h}, 4.083 \mu \mathrm{M}$ at $72 \mathrm{~h}$. At these IC50 dose for $48 \mathrm{~h}$, apoptotic results of oleuropein, vitamin $\mathrm{D}$ and combination treatmentare shown as Figure 1. Oxidative stress index wascalculated by dividing total oxidant capacity by total antioxidant capacity. SOD, GPx, MDA and NO values were calculated by dividing by total protein amount. All biochemical parameters are shown in Figures 2-9.
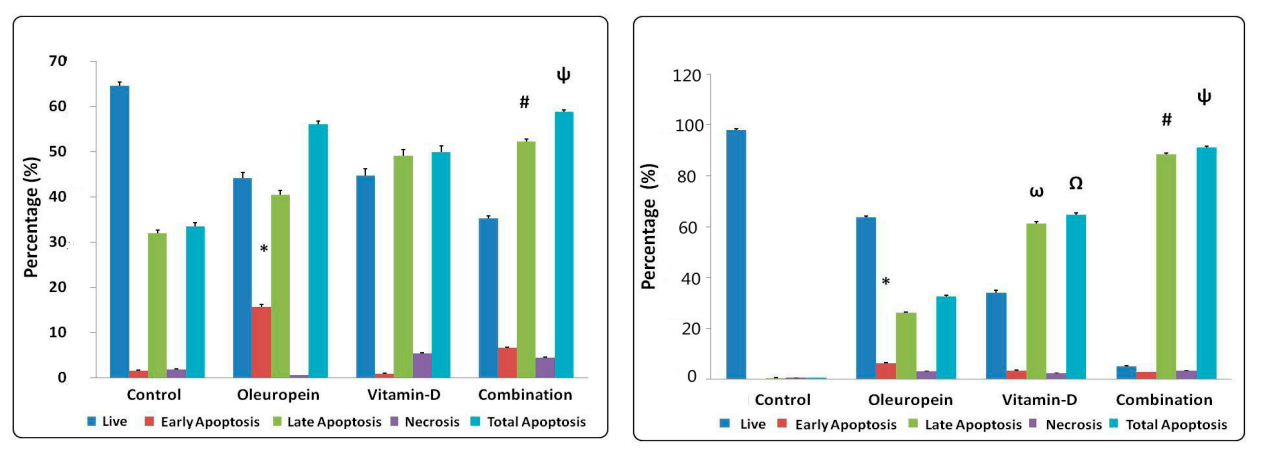

Figure 1. Annexin V and Caspase 3/7analysis results. * Oleuropein treatment has significantly increased early apoptosis as compared to other groups $(p<0.05)$. \# Combination treatment has significantly increased late apoptosis as compared to other groups $(p<0.05)$. $\Psi$ Combination treatment has significantly increased total apoptosis (early + late) as compared to other groups $(p<$ $0.05)$.
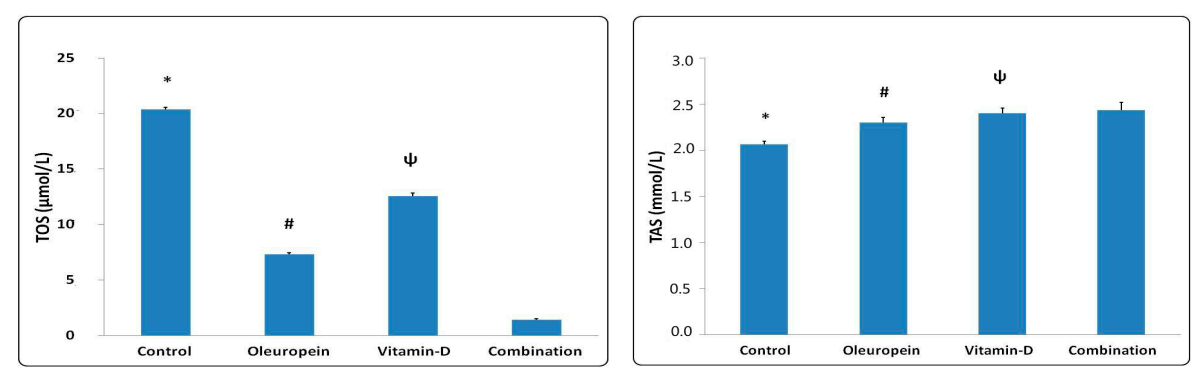


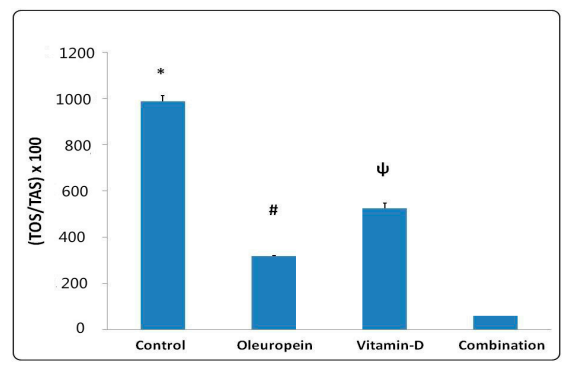

Figure 2. Total oxidant, antioxidant status analysis and oxidative stress index analysis results. \# Oleuropein treatment has significantly decreased OSI as compared to vitamin D treatment $(p<0.05)$. Combination treatment has significantly decreased OSI as compared to oleuropein treatment $(p<0.05)$. $\Psi$ Combination treatment has significantly decreased OSI as compared to vitamin D treatment $(p<0.05)$.

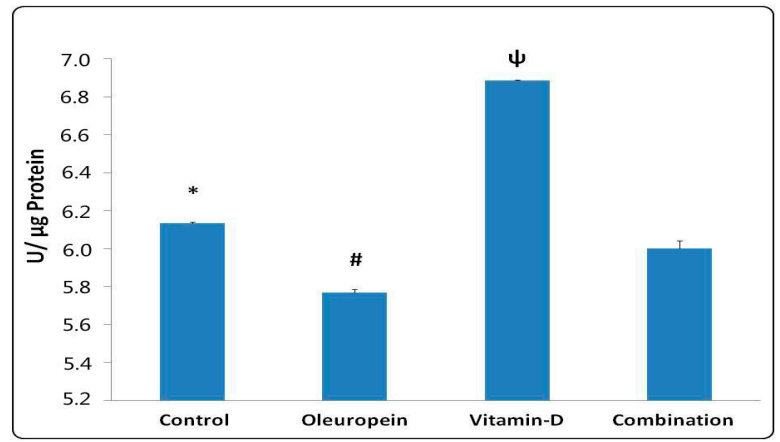

Figure 3. Superoxide dismutase analysis results. $\Psi$ Vitamin D treatment has significantly increased SOD activity as compared to combination treatment group $(p<0.05)$.

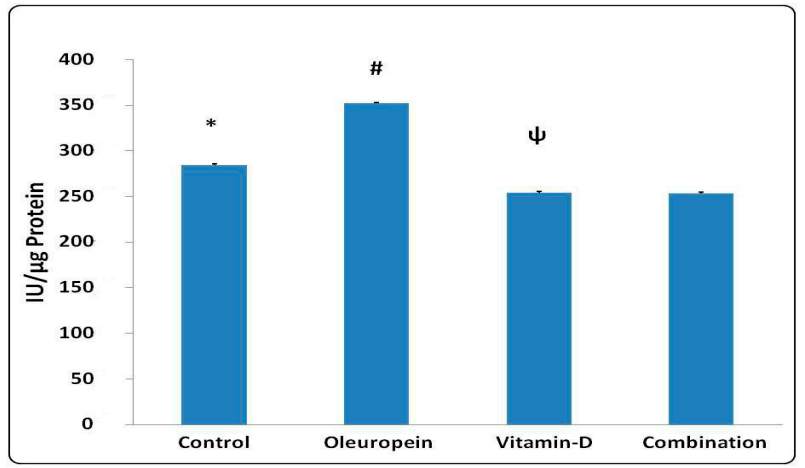

Figure 4. Glutathione peroxidase analysis results. \# Oleuropein treatment has significantly increased GPx as compared to vitamin D and combination treatment.

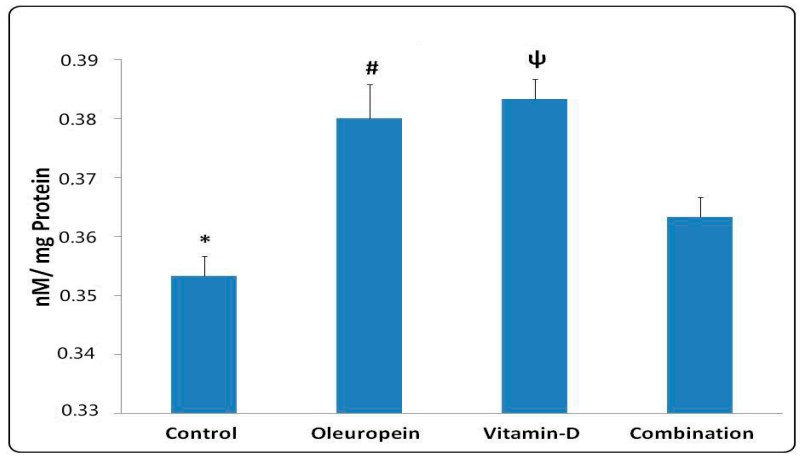

Figure 5. Malondialdehyde analysis results. * Oleuropein and vitamin D treatment has significantly increased the MDA as compared to the control group $(p>0.05)$. 


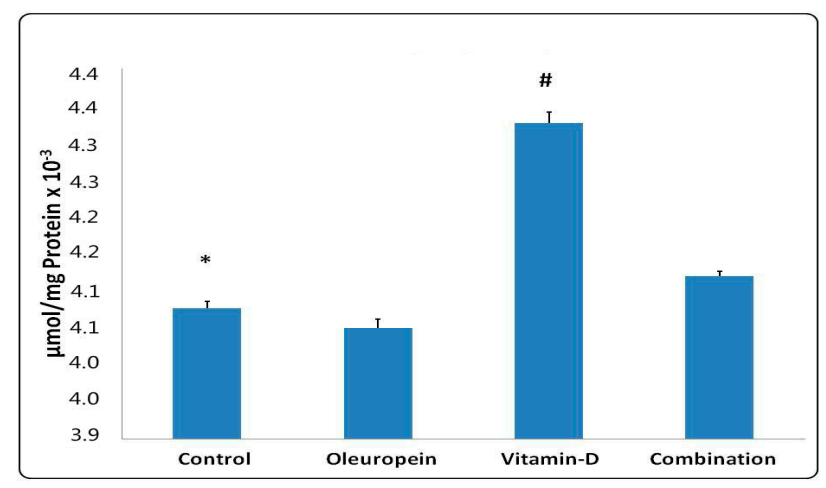

Figure 6. Nitric oxide analysis results. \# Vitamin D treatment has significantly increased NO as compared to other groups $(p<0.05)$.

\section{Discussion}

While chemotherapeutic agents destroy neoplastic cells, some normal cells are also affected. Oleuropein has a strong antioxidant effect, which is especially important in reducing side effects of drugs used in cancer treatments and reducing reactive oxygen species (ROS) levels [4]. Recent studies have revealed that vitamin D modulates breast cancer cell growth and epidemiologic studies suggest increasing vitamin D may be associated with reduced breast cancer risk. Many studies have showed that vitamin $\mathrm{D}$ has an antioxidant effect [5].

Elamin et al. (2013) observed that cell proliferation was significantly inhibited and apoptosis induced when they exposed $200 \mu \mathrm{M}$ of oleuropeine [6]. In our study, IC50 dose of oleuropein was calculated $256.1 \mu \mathrm{M}$ at $24 \mathrm{~h}, 247.5 \mu \mathrm{M}$ at $48 \mathrm{~h}, 222.5 \mu \mathrm{M}$ at $72 \mathrm{~h}$. Vitamin D is not only contributes to maintaining the continuity of calcium metabolism, but also has a wide range of pharmacological significant effects [5]. The antiproliferative, apoptotic, antioxidant effect of vitamin $\mathrm{D}$ is among the most recently proposed non-calcytic roles of this compound [5]. Saracligil et al. (2017) observed the antiproliferative activity of vitamin D in MCF-7 breast cancer cell by real-time cell electronic detection system. IC50 dose of vitamin D was calculated $145 \mathrm{nM}$ [7]. In our study the antiproliferative activity WST-1 test in a wide concentration range of $0.98,1.95,3.906,7.8125,15.62,31.25,62.50,125,250,500$, $1000 \mathrm{nM}$ was studied. Unfortunately no significant inhibition was observed. Murray et al. (2017) the effect of MCF-7 cell line on calcitriol was evaluated by MTT test and the IC50 value was calculated $1.83 \pm 0.69 \mu \mathrm{M}$ [8]. In our study, based on the study of Murray et al. (2017) worked at the range of concentration was $0.25,0.5,1,2,3,4,6,8,12 \mu \mathrm{M}$. IC50 dose of vitamin D was calculated $2.053 \mu \mathrm{M}$ at $48 \mathrm{~h}, 4.083 \mu \mathrm{M}$ at $72 \mathrm{~h}$. In our study, in parallel with many studies, oleuropein and vitamin D showed an antioxidant effect in breast cancer cell line and decreased OSI. Combination treatment has a strong synergetic effect. Oleuropein and vitamin D treatment has significantly increased MDA as compared to control group $(p<0.05)$. After taking the treatment with oleuropein and vitamin $\mathrm{D}$, the cell may have significantly increased lipid peroxidation when it enters oxidative stress and apoptosis. Therefore, we think that the cell may have increased MDA while entering stress. Oleuropein treatment has significantly decreased SOD $(p<0.05)$. Althougholeuropein has a strong antioxidant effect, it has decreased SOD. Antioxidant effect of oleuropein may be via GPx rather than SOD. Vitamin D induces the expression of several molecules involved in the antioxidant defense system. Parallel to this, vitamin D treatment has significantly increased SOD activity $(p<0.05)$. Oleuropeint has significantly increased GPxas compared to other groups $(p<0.05)$. According to this result, oleuropein has a strong antioxidant effect on breast cancer cell line. Vitamin D treatment has significantly increased NO levels ascompared to control group $(p<0.05)$. Vitamin D treatment has a strong cytotoxic effect on MCF-7.

\section{Conclusions}

We have observed that oleuropein and vitamin D reduces cancer cell proliferation depending on dose and time. At the same time, apoptosis increases explain this inhibition of proliferation. The 
increase of antioxidant parameters has been supporting the proliferation inhibiton and induction of apoptosis. We also observed that the combined use of oleuropein and vitamin D is a strong synergetic effect.

Funding: We thank Aydın Adnan Menderes University Scientific Research Projects (BAP). This study was supported by the grants from "Aydın Adnan Menderes University Scientific Research Projects Support Program" (BAP project number) TPF-17035.

\section{References}

1. Saip, P.; Keskin, S.; Ozkan, M.; Kaplan, M.A.; Aydoğan, F.; Demirăg, G.G.; Uzunoğlu, S.; Engin, H.; Başaran, G.; Güler, N.; et al. Türkiye'de meme kanserlihastalarıntanıvetedaviyöntemlerineulaşımhızı; çokmerkezligözlemselçalışma. J. Breast Health 2011, 7, 109-117.

2. Bouaziz, M.; Hammami, H.; Bouallagui, Z.; Jemai, H.; Sayadi, S. Production of antioxidants from olive processing by-products. Electron. J. Environ. Agric. Food Chem. 2008, 7, 3231-3236.

3. Rusinska, A.; Pludowski, P.; Walczak, M.; Borszewska-Kornacka, M.; Bossowski, A.; Chalebna-Sokol, D. Vitamin D supplementation guidelines for general population and groups at risk of vitamin D deficiency in Poland-Recomendations of the Polish society of pediatric endocrinology and diabetes and the expert panel with the participation of the national specialist consultans and representatives of scientific societies2018 update. Front. Endocrinol. 2018, 9, 246.

4. Duijts, S.F.A.; Faber, M.M.; Oldenburg, H.S.A.; Beurden, M.; Aaronson, N.K. Effectiveness of behavioral techniques and physical exercise on psychosocial functioning and health-related quality of life in breast cancer patients and survivors-a meta-analysis. Psycho-Oncology 2011, 20, 115-126.

5. Mokhtari, Z.; Hekmatdoost, A.; Nourian, M. Antioxidant efficacy of vitamin D. J. Parathyroid Dis. 2017, 5, $11-16$.

6. Elamin, M.H.; Daghestani, M.H.; Omer, S.A.; Elobeid, M.A.; Virk, P.; Al-Olayan, E.M.; Hassan, Z.K.; Mohammed, O.B.; Aboussekhra, A. Olive oil oleuropein has anti-breast cancer properties with higher efficiency on ER-negative cells. Food Chem. Toxicol. 2013, 53, 310-316.

7. Saracligil, B.; Ozturk, B.; Unlu, A.; Abusoglu, S.; Tekin, G. The effect of vitamin D on MCF-7 breast cancer cell metabolism. Bratisl Med. J. 2017, 118, 101-106.

8. Murray, A.; Madden, S.F.; Synnott, N.; Klinger, R.; O'Connor, D.; O’Donnovan, N.; Gallagher, W.; Grown, J.; Duffy, M.J. Vitamin D receptor as a target for breast cancer therapy. Soc. Endocrinol. 2017, 24, 181-195. 